Moroccan J. of Pure and Appl. Anal. (MJPAA)

Volume 3(2), 2017, Pages 199-217

ISSN: Online 2351-8227 - Print 2605-6364

DOI 10.1515/mjpaa-2017-0016

\title{
A posteriori error estimates for mixed finite volume solution of elliptic boundary value problems
}

\author{
Fayssal Benkhaldoun $^{1, a}$, Mohammed Seaid $^{2, b}$ And Amadou Mahamane ${ }^{3, c}$
}

\begin{abstract}
The major emphasis of this work is the derivation of a posteriori error estimates for the mixed finite volume discretization of second-order elliptic equations. The estimates are established for meshes consisting of simplices on unstructured grids. We consider diffusion problems with nonhomogeneous diffusion coefficients. The error estimates are of residual types and are formulated in the energy semi-norm for a locally postprocessed approximate solutions. The estimates are fully computable and locally efficient that they can serve as indicators for adaptive refinement and for the actual control of the error. Numerical results are shown for two test examples in two space dimensions. It is found that the proposed adaptive mixed finite volume method offers a robust and accurate approach for solving second-order elliptic equations, even when highly nonhomogeneous diffusion coefficients are used in the simulations.
\end{abstract}

2010 Mathematics Subject Classification. 35J15, 65M08, 65L20, 65N50.

Key words and phrases. .A posteriori error estimate; mixed finite volume methods; second-order elliptic equations; unstructured mesh; diffusion equations

\section{Introduction}

Second-order elliptic problems with discontinuous coefficients arise in mathematical modeling processes in diffusion in composite media, heat and mass transfer, flows in porous media, among others. In many applications

Received June 12018 - Accepted June 25, 2018, published online in July 2018.

(C)The Author(s) 2017. This article is published with open access by Sidi Mohamed Ben Abdallah University.

${ }^{1}$ Université Paris 13, Sorbonne Paris Cité, LAGA, CNRS (UMR 7539) 99 Av J.B. Clement, 93430 Villetaneuse

a e-mail: fayssal@math.univ-paris13.fr

${ }^{2}$ Laboratory of Complex Systems Engineering 83 Human Systems, University Mohammed VI Polytechnic, 43150 Benguerir, Morocco and School of Engineering and Computing Sciences, University of Durham, South Road, Durham DH1 3LE, UK

${ }^{b}$ e-mail: m.seaid@durham.ac.uk

${ }^{3}$ Université des sciences, des techniques et des technologies de Bamako, Mali

${ }^{c}$ e-mail: moulaye.ahmad@gmail.com . 
for these problems, the data describing the properties of the medium (conductivity, permeability, porosity) involve high discontinuities or numerous heterogeneities. Therefore, elliptic equations with discontinuous coefficients impose great challenges to the numerical methods. In general, to solve numerically such equations, we commonly use finite element or finite volume methods. There are several highly desirable properties of the discretization beyond the classical stability and accuracy, such as local mass conservation, discrete maximum principle, etc, that are crucial in capturing the important details of the solutions of complicated problems on relatively coarse grids. Then, if we work with meshes adapted to the spatial distribution of the coefficients, we obtain a system of large size, that is prohibitively expensive to solve. In order to avoid this, we would want to use any reasonable structured mesh. Thereby, some elements of the mesh will be crossed by the coefficients discontinuity surfaces and this implies to replace on these elements the true coefficient by coefficients that generate nearly equivalent description of the physical properties.

Finite volume methods are well adapted to the discretization of conservation laws, see for example [18]. These methods provide efficient numerical schemes for elliptic equations as well as for convection-dominated parabolic equations. However, because of the heterogeneous coefficients in the considered equations, the standard four-point finite volume schemes cannot be used here. Another class of finite volumes schemes for solving diffusion equations on two- and three-dimensional unstructured grids has been developed in [14]. Other finite volume methods for solving diffusion equations have also been studied in the literature such as Discrete Duality Finite Volume (DDFV) method [14, 8] and Multipoint Flux Approximation (MPFA) [1] method among others. The DDFV method also arises from the construction of discrete analogues of the divergence and flux operators which fulfill the discrete counterpart of vector calculus identities. However, this method requires to solve the diffusion equation not only over the primal grid but also over a dual grid. Namely, there are both cell-centered and vertex-centered unknowns. Compared to a classical cell-centered finite volume scheme, the DDFV method necessitates twice as much degrees of freedom over quadrangular grids. In [10], a mixed finite volume method is proposed which handles heterogeneous diffusion problems on any grid and precisely provides good approximations of the problem. This method is suited for unstructured grids and adaptive grid refinement as well as for efficient parallelization by domain decomposition. This method has been successfully applied to convection-diffusion-reaction equations in $[15,4]$. In the current study, we shall analyze a discretization of second-order elliptic equations based on a mixed finite volume method. Essentially, this approach consists of writing the considered problem in the mixed form, and discretizing the flux variable using finite volume techniques, and the scalar variable by piecewise constants. Throughout this work, for simplicity we restrict our attention to triangular elements, and the extension to three-dimensional problems is straightforward.

As for most mesh-based methods for solving partial differential equations, the computational cost in finite volume methods becomes more and more important (sometimes prohibitive) when we need numerical solution with good accuracy. It is also well known that a posteriori error analysis is one approach to find a compromise between cost and accuracy of these methods. The importance of a posteriori error estimates for controlling an automatic, self-adaptive mesh-refinement process is well established in recent decades; see for instance [3, 20] for extensive reviews. Deriving a posteriori error estimates for finite volume approximations of the elliptic equations also has received much attention. The work of Verfurth et al. [20] builds the basic foundation for the mathematical analysis of practical methods and has been advanced by Vohralík et al. [21, 22] and others. In these works, the authors present a general framework for a posteriori error analysis for finite volume methods applied to convection-diffusion-reaction problems. A posteriori error estimates for finite volume methods have also derived in $[16,6,13,5]$ for the standard two-point finite volume schemes, in [17, 2] for DDFV schemes, and in [7] for mimetic finite difference methods. In most of the works mentioned above, the derivation of a posteriori error estimators needs to construct a novel solution which is more regular than the one produced by the scheme. Sometimes this procedure is very expensive. In this work we derive in a very cheap way a posteriori error estimators for the mixed finite volume scheme applied to elliptic equations. The proposed method does not resolve any local linear systems and it consists of an explicit reconstruction of the postprocessed solution using the nodal values evaluated by the method. Recently, the mixed finite volume methods, the mimetic finite difference methods, the hybrid finite volume methods and mixed finite elements have been found to be equivalent [11]. This finding supports the fact that the analysis presented in this study remains valid for these methods. In this paper we derive a posteriori error estimates for the mixed finite volume discretization of second-order elliptic equations. The natural combination of adaptive techniques with mixed finite volume methods retains the best features of both methods and overcomes many of their defects. Their implementation relies on the 
projection operators, whose actions use only standard nodal data structures and can be evaluated locally at the element level. Additionally, the combination turns out to be particularly attractive on modern parallel processing architectures. Finally, by assuming the nondegeneracy property, we can obtain local lower and upper bounds for the error of the numerical solution. We numerically illustrate the efficiency of our techniques by solving two test examples for second-order elliptic problems with discontinuous coefficients.

The structure of the paper is a follows. In section 2 we collect some notations and preliminaries. The mixed finite volume method is formulated in section 3. This section includes the finite volume discretization and the formulation of the mixed finite volume method for the considered elliptic problem. In section 4 we present a posterior error estimates for the proposed finite volume method. Section 5 contains numerical results for two test examples on diffusion equations. Concluding remarks are given in section 6 .

\section{Preliminaries and Notations}

We consider second-order elliptic equations with heterogeneous coefficients. For this class of problems, the diffusion in a two-dimensional closed medium $\Omega \subset \mathbb{R}^{2}$ with boundary $\partial \Omega$ is described by the following equation

$$
\begin{array}{rlrl}
-\nabla \cdot(\kappa(\mathbf{x}) \nabla u(\mathbf{x})) & =f(\mathbf{x}), & \forall \mathbf{x} \in \Omega, \\
u(\mathbf{x}) & =0, \quad \forall \mathbf{x} \in \partial \Omega,
\end{array}
$$

where $f \in L^{2}(\Omega)$ is the source term and $\kappa$ is the diffusion coefficient assumed to be piecewise constant such that there exist two positive constants $\underline{\kappa}$ and $\bar{\kappa}$ for which

$$
0<\underline{\kappa} \leq \kappa(\mathbf{x}) \leq \bar{\kappa}, \quad \text { a.e. } \quad \mathbf{x} \in \Omega .
$$

The spatial domain $\bar{\Omega}=\Omega \cup \partial \Omega$ is discretized in an admissible finite volume discretization $\mathcal{D}=(\mathcal{M}, \mathcal{E}, \mathcal{P})$, where $\mathcal{M}$ is the set of control volumes $\bar{K}$ with $\bar{\Omega}=\bigcup_{K \in \mathcal{M}} \bar{K}, \mathcal{E}$ is the finite set of mesh interfaces, and $\mathcal{P}=\left(\mathbf{x}_{K}\right)_{K \in \mathcal{M}}$ are the mesh points in $K$ for each $K \in \mathcal{M}$. We denote by $\mathcal{S}$ the set of all nodes $P$ in the mesh and by $\mathcal{V}_{P}$ the set of all cells $K \in \mathcal{M}$ for which $P$ is a vertex. We assume that there exists a subset $\mathcal{E}_{K} \subset \mathcal{E}$ such that $\partial K=\cup_{\sigma \in \mathcal{E}_{K}} \bar{\sigma}$, for each $\sigma \in \mathcal{E}_{K}$, and $\mathbf{n}_{K, \sigma}$ denotes the unit normal vector to $\sigma$ outward to $K$. Furthermore, we assume that for $\sigma \in \mathcal{E}$ and for a pair $(K, L) \in \mathcal{M}^{2}$, either $\sigma \in \partial \Omega$ or $\bar{\sigma}=\bar{K} \cap \bar{L}$ and in the later case we use the notation $\sigma=K \mid L$. We also use the notation $\mathrm{m}(\mathrm{K})$ and $\mathrm{m}(\sigma)$ to denote the area of $K$ and the length of $\sigma$, respectively. Here $h_{K}$ is the diameter of the cell $K$ and $h=\max _{K \in \mathcal{M}} h_{K}$ is the mesh size.

We define the discrete functional space $H_{\mathcal{M}}$ and $P^{2}(\mathcal{M})$ as the set of constant functions in each $K \in \mathcal{M}$ and the set of polynomials of total degree at most 2 in each cell $K$, respectively. The discrete $\operatorname{Sobolev}$ space $H^{1}(\mathcal{M})$ is defined by

$$
H^{1}(\mathcal{M})=\left\{\varphi \in L^{2}(\Omega):\left.\quad \varphi\right|_{K} \in H^{1}(K), \quad \forall K \in \mathcal{M}\right\} .
$$

We also introduce the bilinear form $a(\cdot, \cdot)$ defined in $H^{1}(\mathcal{M}) \times H^{1}(\mathcal{M})$ as

$$
a(v, w)=\sum_{K \in \mathcal{M}} \int_{K} \kappa \nabla v \cdot \nabla w d \mathbf{x}
$$

and the associated energy-norm ||$|\cdot|||$ defined as

$$
\|u\|\left\|^{2}=\sum_{K \in \mathcal{M}}\right\|\|u\|_{K}^{2}
$$

where

$$
\|u\|_{K}^{2}=\int_{K} \kappa \nabla u \cdot \nabla u d \mathbf{x} .
$$

For each $\left(u_{h}, \mathbf{v}_{h}\right) \in H_{\mathcal{M}} \times H_{\mathcal{M}}^{2}$, we define the following discrete norms

$$
\left\|u_{h}\right\|_{L^{2}(\Omega)}^{2}=\sum_{K \in \mathcal{M}} \mathrm{m}(K) u_{K}^{2}, \quad\left\|\mathbf{v}_{h}\right\|_{L^{2}(\Omega)^{2}}^{2}=\sum_{K \in \mathcal{M}} \mathrm{m}(K)\left|\mathbf{v}_{K}\right|_{2}^{2},
$$


where $|\cdot|_{2}$ denotes the canonical Euclidean norm in $\mathbb{R}^{2}$. We define $F=\left(F_{K, \sigma}\right)_{\sigma \in \mathcal{E}_{K}, K \in \mathcal{M}}$ as a family of real numbers which approximate the flux $\int_{\sigma} \kappa \nabla u \cdot \mathbf{n}_{K, \sigma}$ ds and $\mathcal{F}_{\mathcal{M}}$ is the set of such families.

For a given family $\left(\nu=\left(\nu_{K}\right)_{K \in \mathcal{M}}\right)$ of positive real numbers, we define the set $L_{\nu}(\mathcal{M})$ of triplets $\left(u_{h}, \mathbf{v}_{h}, F\right) \in$ $H_{\mathcal{M}} \times H_{\mathcal{M}}^{2} \times \mathcal{F}_{\mathcal{M}}$ such that

$$
\begin{aligned}
\mathbf{v}_{K} \cdot\left(\mathbf{x}_{\sigma}-\mathbf{x}_{K}\right)+\mathbf{v}_{L} \cdot\left(\mathbf{x}_{L}-\mathbf{x}_{\sigma}\right)+\nu_{K} \mathrm{~m}(K) F_{K, \sigma}- & \\
\nu_{L} \mathrm{~m}(L) F_{L, \sigma}=u_{L}-u_{K}, & \forall \sigma=K \mid L \in \mathcal{E}_{\text {int }}, \\
\mathbf{v}_{K} \cdot\left(\mathbf{x}_{\sigma}-\mathbf{x}_{K}\right)+\nu_{K} \mathrm{~m}(K) F_{K, \sigma}=-u_{K}, & \forall \sigma \in \mathcal{E}_{K} \cap \mathcal{E}_{\text {ext }} .
\end{aligned}
$$

In the sequel, unless otherwise stated, $C$ denotes a positive constant independent of the mesh size $h$.

\section{Mixed Finite Volume Methods}

Applied to the problem (1) a mixed finite volume method yields the statement of the problem: find $(u, \mathbf{v}, F) \in$ $L_{\nu}(\mathcal{M})$ such that

$$
\begin{array}{rlrl}
F_{K, \sigma}+F_{L, \sigma} & =0, & & \forall \sigma=K \mid L \in \mathcal{E}_{\text {int }}, \\
\mathrm{m}(K) \mathbf{v}_{K}=\sum_{\sigma \in \mathcal{E}_{K}} F_{K, \sigma} \kappa_{K}^{-1}\left(\mathbf{x}_{\sigma}-\mathbf{x}_{K}\right), & & \forall K \in \mathcal{M}, \\
-\sum_{\sigma \in \mathcal{E}_{K}} F_{K, \sigma}=\mathrm{m}(K) f_{K}, & & \forall K \in \mathcal{M},
\end{array}
$$

where $f_{K}=\frac{1}{\mathrm{~m}(K)} \int_{K} f(\mathbf{x}) d \mathbf{x}$. It should be stressed that the mixed finite volume method (3) employs the same techniques used in the literature for mixed finite element methods and the implementation procedure of this class of mixed finite volume methods can be carried out as in [9] among others. Thus, for each solution $\left(u_{h}, \mathbf{v}_{h}, F\right)$ fulfilling the relations (2) and (3b) we define the unknowns $\left(u_{\sigma}\right)_{\sigma \in \mathcal{E}}$ as

$$
\mathbf{v}_{K} \cdot\left(\mathbf{x}_{\sigma}-\mathbf{x}_{K}\right)+\nu_{K} \mathrm{~m}(K) F_{K, \sigma}=u_{\sigma}-u_{K}, \quad \forall K \in \mathcal{M}, \quad \forall \sigma \in \mathcal{E}_{K}
$$

with $u_{\sigma}=0$ when $\sigma \in \mathcal{E}_{\text {ext }}$. The equations (3b) and (4) yield

$$
\begin{aligned}
\frac{1}{\mathrm{~m}(\mathrm{~K})} \sum_{\sigma^{\prime} \in \mathcal{E}_{K}} F_{K, \sigma^{\prime}} \Lambda_{K}^{-1}\left(\mathbf{x}_{\sigma^{\prime}}-\mathbf{x}_{K}\right) \cdot\left(\mathbf{x}_{\sigma}-\mathbf{x}_{K}\right)+ \\
\nu_{K} \mathrm{~m}(K) F_{K, \sigma}=u_{\sigma}-u_{K}, \quad \forall K \in \mathcal{M}, \quad \forall \sigma \in \mathcal{E}_{K} .
\end{aligned}
$$

Hence, for each $K \in \mathcal{M}$, the above formulation leads to a linear system of algebraic equations to be solved for the unknowns $\left(F_{K, \sigma}\right)_{\sigma \in \mathcal{E}_{K}}$ as

$$
A_{K}\left(F_{K, \sigma}\right)_{\sigma \in \mathcal{E}_{K}}=\left(u_{\sigma}-u_{K}\right)_{\sigma \in \mathcal{E}_{K}} .
$$

Note that, due to the condition $\nu_{K}>0$, the matrix $A_{K}$ is symmetric and positive definite. In addition, the solution of the linear system can be reformulated as

$$
F_{K, \sigma}=\sum_{\sigma^{\prime} \in \mathcal{E}_{K}}\left(A_{K}^{-1}\right)_{\sigma \sigma^{\prime}}\left(u_{\sigma^{\prime}}-u_{K}\right), \quad \forall K \in \mathcal{M}, \quad \forall \sigma \in \mathcal{E}_{K} .
$$

Using the relation (3c), it is easy to verify

$$
-\sum_{\sigma^{\prime} \in \mathcal{E}_{K}} \beta_{K, \sigma^{\prime}} u_{\sigma^{\prime}}+\beta_{K} u_{K}=\int_{K} f(\mathbf{x}) d \mathbf{x}
$$

where

$$
\beta_{K, \sigma^{\prime}}=\sum_{\sigma \in \mathcal{E}_{K}}\left(A_{K}^{-1}\right)_{\sigma \sigma^{\prime}}, \quad \beta_{K}=\sum_{\sigma^{\prime} \in \mathcal{E}_{K}} \beta_{K, \sigma^{\prime}} .
$$


Let $(1)_{\sigma^{\prime} \in \mathcal{E}_{K}}$ denotes the vector in $\mathbb{R}^{\operatorname{Card}\left(\mathcal{E}_{\mathrm{K}}\right)}$ with all its components are equal to 1 , with $\operatorname{Card}\left(\mathcal{E}_{\mathrm{K}}\right)$ denotes the cardinality of the set $\mathcal{E}_{K}$. Using the property

$$
\left(\beta_{K, \sigma^{\prime}}\right)_{\sigma^{\prime} \in \mathcal{E}_{K}}=A_{K}^{-1}(1)_{\sigma^{\prime} \in \mathcal{E}_{K}},
$$

one obtains

$$
\beta_{K}=(1)_{\sigma^{\prime} \in \mathcal{E}_{K}} \cdot A_{K}^{-1}(1)_{\sigma^{\prime} \in \mathcal{E}_{K}} .
$$

Since the matrix $A_{K}^{-1}$ is symmetric and positive definite, it follows

$$
\beta_{K}>0, \quad \forall K \in \mathcal{M} .
$$

Thus, the solution $u_{K}$ is given by

$$
u_{K}=\frac{1}{\beta_{K}}\left(\sum_{\sigma^{\prime} \in \mathcal{E}_{K}} \beta_{K, \sigma^{\prime}} u_{\sigma^{\prime}}+\int_{K} f(\mathbf{x}) d \mathbf{x}\right) .
$$

Using the relation (3a) along with the formulation (6), we obtain

$$
\begin{aligned}
\sum_{\sigma^{\prime} \in \mathcal{E}_{K}}\left(\left(A_{K}^{-1}\right)_{\sigma \sigma^{\prime}}-\frac{\beta_{K, \sigma} \beta_{K, \sigma^{\prime}}}{\beta_{K}}\right) & u_{\sigma^{\prime}}+\sum_{\sigma^{\prime} \in \mathcal{E}_{L}}\left(\left(A_{L}^{-1}\right)_{\sigma \sigma^{\prime}}-\frac{\beta_{L, \sigma} \beta_{L, \sigma^{\prime}}}{\beta_{L}}\right) u_{\sigma^{\prime}} \\
= & \frac{\beta_{K, \sigma}}{\beta_{K}} \int_{K} f(\mathbf{x}) d \mathbf{x}+\frac{\beta_{L, \sigma}}{\beta_{L}} \int_{L} f(\mathbf{x}) d \mathbf{x}, \quad \forall \sigma=K \mid L \in \mathcal{E}_{\text {int }} .
\end{aligned}
$$

It is worth remarking that this linear system has been studied in [9] for which the matrix is proven to be symmetric and positive definite. Thus, solving the linear system $(7)$, the computation of $\left(u_{h}, F\right)$ is performed using the relations (6) and (5), whereas $\mathbf{v}_{h}$ is calculated using the relation (3b).

\section{A Posterior Error Estimates}

In this section we present a posterior error estimates for the mixed finite volume method (3). It should be mentioned that a priori error estimates for this class of finite volume methods can be found for instance in [9]. Hence, we present the following results:

Lemma 4.1. If the mesh $\mathcal{M}$ is formed using simplex then the finite volume approximation $\left(u_{h}, \mathbf{v}_{h}\right)$ of the solution $(u, \nabla u)$ satisfies the following inequalities:

$$
\begin{aligned}
\left\|u-u_{h}\right\|_{L^{2}(\Omega)} & \leq C h, \\
\left\|\nabla u-\mathbf{v}_{h}\right\|_{L^{2}(\Omega)^{2}} & \leq C h .
\end{aligned}
$$

For the proof of this lemma we refer to [9, Theorem 2.3, Lemma 6.4].

To construct the error estimates we first consider a postprocessed solution denoted by $\tilde{u}_{h}$ and then we proceed using similar steps of the analysis presented in [22]. This consists on finding $\tilde{u}_{h}$ as a weak solution of the following local Neumann problems:

$$
\begin{aligned}
-\nabla \cdot\left(\kappa_{K} \nabla \tilde{u}_{h}\right) & =\frac{1}{\mathrm{~m}(K)} \sum_{\sigma \in \mathcal{E}_{K}}-F_{K, \sigma}, & & \forall K \in \mathcal{M}, \\
\tilde{u}_{h}\left(\mathbf{x}_{K}\right) & =u_{K}, & & \forall K, \in \mathcal{M}, \\
-\left.\kappa_{K} \nabla \tilde{u}_{h}\right|_{K} \cdot \mathbf{n}_{K, \sigma} & =-\frac{F_{K, \sigma}}{\mathrm{m}(\sigma)}, & & \forall \sigma \in \mathcal{E}_{K}, \quad \forall K \in \mathcal{M},
\end{aligned}
$$

where $u_{K}$ is the approximation of $u_{h}$ in the control volume $K, F_{K, \sigma}$ is the approximated flux at the surface $\sigma$ of the element $K$. Note that similar estimations are presented in [22] on conform meshes formed of simplices for second-order polynomial solutions $\tilde{u}_{h}$. 


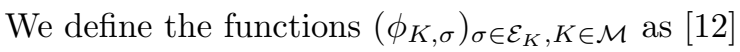

$$
\begin{array}{rlrl}
\Delta \phi_{K, \sigma}(\mathbf{x}) & =\frac{\mathrm{m}(\sigma)}{\mathrm{m}(K)}, & & \text { a.e. } \mathbf{x} \in K, \\
\int_{K} \phi_{K, \sigma}(\mathbf{x}) d \mathbf{x} & =0, & & \text { a.e. } \mathbf{y} \in \sigma, \\
\nabla \phi_{K, \sigma}(\mathbf{y}) \cdot \mathbf{n}_{K, \sigma} & =1, & & \text { a.e. } \mathbf{y} \in \tilde{\sigma}, \quad \tilde{\sigma} \in \mathcal{E}_{K}, \quad \tilde{\sigma} \neq \sigma . \\
\nabla \phi_{K, \sigma}(\mathbf{y}) \cdot \mathbf{n}_{K, \tilde{\sigma}} & =0, \quad
\end{array}
$$

We also define the coefficients $\alpha_{K, \sigma}$ as

$$
\alpha_{K, \sigma}:=\left(\kappa_{K}^{-1} \frac{F_{K, \sigma}}{\mathrm{m}(\sigma)}-\mathbf{v}_{K} \cdot \mathbf{n}_{K, \sigma}\right), \quad \forall \sigma \in \mathcal{E}_{K}, \quad \forall K \in \mathcal{M} .
$$

Lemma 4.2. Assume a triangular mesh $\mathcal{M}$ and $\left(u_{h}, \mathbf{v}_{h}, F\right)$ is the solution of the problem (3). The solution $\tilde{u}_{h}$ of the local problems (10) is given by

$$
\left.\tilde{u}_{h}\right|_{K}(\mathbf{x})=u_{K}+\mathbf{v}_{K} \cdot\left(\mathbf{x}-\mathbf{x}_{K}\right)+\sum_{\sigma \in \mathcal{E}_{K}} \alpha_{K, \sigma}\left(\phi_{K, \sigma}(\mathbf{x})-\phi_{K, \sigma}\left(\mathbf{x}_{K}\right)\right), \quad \forall K \in \mathcal{M} .
$$

Proof. By definition of the solution $\tilde{u}_{h}$, it satisfies the equation (10b). From (11) we obtain

$$
\left.\nabla \tilde{u}\right|_{K}=\mathbf{v}_{K}+\sum_{\sigma \in \mathcal{E}_{K}} \alpha_{K, \sigma} \nabla \phi_{K, \sigma}, \quad \forall K \in \mathcal{M} .
$$

Hence, for each $\sigma_{0} \in \mathcal{E}_{K}$

$$
-\left.\kappa_{K} \nabla \tilde{u}_{h}\right|_{K} \cdot \mathbf{n}_{K, \sigma_{0}}=\left(-\kappa_{K} \mathbf{v}_{K}-\kappa_{K} \sum_{\sigma \in \mathcal{E}_{K}} \alpha_{K, \sigma} \nabla \phi_{K, \sigma}\right) \cdot \mathbf{n}_{K, \sigma_{0}} .
$$

Using the definition of the functions $\phi_{K, \sigma}$ and the coefficients $\alpha_{K, \sigma}$, we obtain

$$
-\left.\kappa_{K} \nabla \tilde{u}_{h}\right|_{K} \cdot \mathbf{n}_{K, \sigma_{0}}=-\frac{F_{K, \sigma_{0}}}{\mathrm{~m}\left(\sigma_{0}\right)},
$$

and therefore, $\tilde{u}_{h}$ solves the equation (10c). Consequently, $\tilde{u}_{h}$ solves the equation (10a) and this resumes the proof.

Lemma 4.3. Under the assumptions of Lemma 4.2, the solution $\tilde{u}_{h} \in P^{2}(\mathcal{M})$ and $\left.\tilde{u}_{h}\right|_{K}(\mathbf{x})$ is given by

$$
\left.\tilde{u}_{h}\right|_{K}(\mathbf{x})=a_{K}\left(x^{2}+y^{2}\right)+b_{K} x+c_{K} y+d_{K}, \quad \forall K \in \mathcal{M},
$$

where

$$
a_{K}=-\frac{1}{4 \kappa_{K}} f_{K} .
$$

Proof. From the analysis reported in [12] it is known that for triangular elements

$$
\nabla \phi_{K, \sigma}(\mathbf{x})=\frac{\mathbf{x}-\mathbf{a}_{\sigma}}{h_{\sigma}},
$$

where $\mathbf{a}_{\sigma}$ is a nodal point in the triangle $K$ opposite to the face $\sigma$, and $h_{\sigma}$ is the distance between $\mathbf{a}_{\sigma}$ and $\sigma$. Hence, $\phi_{K, \sigma}$ is a second-order polynomial of the following form

$$
a_{K, \sigma}\left(x^{2}+y^{2}\right)+b_{K, \sigma} x+c_{K, \sigma} y+d_{K, \sigma},
$$

and $\left.\tilde{u}_{h}\right|_{K}(\mathbf{x})$ is of the required form (12). Next we show that $a_{K}$ satisfies the equation (13). From the definition of $\left.\tilde{u}_{h}\right|_{K}(\mathbf{x})$ we have

$$
\left.\Delta \tilde{u}_{h}\right|_{K}=4 a_{K} .
$$


Using the definition of $\left.\tilde{u}_{h}\right|_{K}$ in (11) yields

$$
\left.\Delta \tilde{u}_{h}\right|_{K}=\sum_{\sigma \in \mathcal{E}_{K}} \alpha_{K, \sigma} \Delta \phi_{K, \sigma}
$$

By substituting the expressions of $\alpha_{K, \sigma}$ and $\Delta \phi_{K, \sigma}$ one obtains

$$
4 a_{K}=\kappa_{K}^{-1} \frac{1}{\mathrm{~m}(\mathrm{~K})} \sum_{\sigma \in \mathcal{E}_{K}} F_{K, \sigma}-\frac{1}{\mathrm{~m}(\mathrm{~K})} \mathbf{v}_{K} \cdot\left(\sum_{\sigma \in \mathcal{E}_{K}} \mathrm{~m}(\sigma) \mathbf{n}_{K, \sigma}\right) .
$$

Since the mesh $\mathcal{M}$ is assumed to be triangular

$$
\sum_{\sigma \in \mathcal{E}_{K}} \mathrm{~m}(\sigma) \mathbf{n}_{K, \sigma}=0, \quad \forall K \in \mathcal{M} .
$$

The relation (13) holds following the equation (3c) from the definition of the considered mixed finite volume method.

Lemma 4.4. If the nodal points $\left(\mathbf{x}_{K}\right)_{K \in \mathcal{M}}$ are selected such that each $K \in \mathcal{M}$, $\mathbf{x}_{K}$ is the isobarycentre of the element $K$, then

$$
\nabla \tilde{u}_{h}\left(\mathbf{x}_{K}\right)=\mathbf{v}_{K}, \quad \forall K \in \mathcal{M}
$$

Proof. From (11) we get

$$
\nabla \tilde{u}_{h}\left(\mathbf{x}_{K}\right)=\mathbf{v}_{K}+\sum_{\sigma \in \mathcal{E}_{K}} \alpha_{K, \sigma} \nabla \phi_{K, \sigma}\left(\mathbf{x}_{K}\right) .
$$

By substituting $\alpha_{K, \sigma}$ and $\Delta \phi_{K, \sigma}$ using their associated expressions one obtains

$$
\nabla \tilde{u}_{h}\left(\mathbf{x}_{K}\right)=\mathbf{v}_{K}+\sum_{\sigma \in \mathcal{E}_{K}}\left(\kappa_{K}^{-1} \frac{F_{K, \sigma}}{\mathrm{m}(\sigma)}-\mathbf{v}_{K} \cdot \mathbf{n}_{K, \sigma}\right) \frac{\mathbf{x}_{K}-\mathbf{a}_{\sigma}}{h_{\sigma}}
$$

however,

$$
h_{\sigma}=\frac{2 \mathrm{~m}(K)}{\mathrm{m}(\sigma)} \quad \text { and } \quad \mathbf{x}_{K}-\mathbf{a}_{\sigma}=2\left(\mathbf{x}_{\sigma}-\mathbf{x}_{K}\right), \quad \forall \sigma \in \mathcal{E}_{K}
$$

Thus,

$$
\nabla \tilde{u}_{h}\left(\mathbf{x}_{K}\right)=\mathbf{v}_{K}+\frac{1}{\mathrm{~m}(K)} \sum_{\sigma \in \mathcal{E}_{K}} \kappa_{K}^{-1} F_{K, \sigma}\left(\mathbf{x}_{\sigma}-\mathbf{x}_{K}\right)-\frac{1}{\mathrm{~m}(K)} \sum_{\sigma \in \mathcal{E}_{K}} \mathrm{~m}(\sigma) \mathbf{v}_{K} \cdot \mathbf{n}_{K, \sigma}\left(\mathbf{x}_{\sigma}-\mathbf{x}_{K}\right) .
$$

Using the relation $(3 \mathrm{~b})$ we obtain

$$
\mathbf{v}_{K}=\frac{1}{\mathrm{~m}(K)} \sum_{\sigma \in \mathcal{E}_{K}} \kappa_{K}^{-1} F_{K, \sigma}\left(\mathbf{x}_{\sigma}-\mathbf{x}_{K}\right),
$$

and the application of the results from [9, Lemma 6.1] to $\mathbf{v}_{K}$ gives

$$
\mathbf{v}_{K}=\frac{1}{\mathrm{~m}(K)} \sum_{\sigma \in \mathcal{E}_{K}} \mathrm{~m}(\sigma) \mathbf{v}_{K} \cdot \mathbf{n}_{K, \sigma}\left(\mathbf{x}_{\sigma}-\mathbf{x}_{K}\right),
$$

and the proof is achieved.

Lemma 4.5. Let $\left(u_{h}, \mathbf{v}_{h}\right)$ be the approximation of $(u, \nabla u)$ by the proposed mixed finite volume method and let $\tilde{u}_{h}$ be the reconstructed solution in (11). Hence,

$$
\begin{aligned}
\left\|\tilde{u}_{h}-u_{h}\right\|_{L^{2}(\Omega)} & \leq C h, \\
\left\|\nabla \tilde{u}_{h}-\mathbf{v}_{h}\right\|_{L^{2}(\Omega)^{2}} & \leq C h,
\end{aligned}
$$


Proof. Since by definition $\tilde{u}_{h}\left(\mathbf{x}_{K}\right)=u_{K}$ and $\left.\tilde{u}_{h}\right|_{K}$ is a second-order polynomial, we have

$$
\left.\tilde{u}_{h}\right|_{K}(\mathbf{x})-u_{K}=\nabla \tilde{u}_{h}\left(\mathbf{x}_{K}\right) \cdot\left(\mathbf{x}-\mathbf{x}_{K}\right)+\frac{1}{2}\left(\mathbf{x}-\mathbf{x}_{K}\right) \cdot \mathbf{H}\left(\mathbf{x}_{K}\right)\left(\mathbf{x}-\mathbf{x}_{K}\right),
$$

where $\mathbf{H}\left(\mathbf{x}_{K}\right)$ is the Hessian matrix of $\left.\tilde{u}_{h}\right|_{K}$ in $\mathbf{x}_{K}$. Using the form of $\left.\tilde{u}_{h}\right|_{K}$, we obtain

$$
\mathbf{H}(\mathbf{x})=2 a_{K}, \quad \forall \mathbf{x} \in K \text {. }
$$

From Lemma 4.4, it follows that

$$
\tilde{u}_{h}(\mathbf{x})-u_{K}=\mathbf{v}_{K} \cdot\left(\mathbf{x}-\mathbf{x}_{K}\right)+a_{K}\left|\mathbf{x}-\mathbf{x}_{K}\right|_{2}^{2}, \quad \forall \mathbf{x} \in K .
$$

Using the Young inequality $\left(a b \leq \frac{1}{2}\left(a^{2}+b^{2}\right)\right)$ and the Cauchy-Schwartz inequality, we obtain

$$
\left.\left(\left.\tilde{u}_{h}\right|_{K}(\mathbf{x})-u_{K}\right)^{2} \leq\left. 2\left(\left|\mathbf{v}_{K}\right|_{2} \mid \mathbf{x}-\mathbf{x}_{K}\right)\right|_{2}\right)^{2}+2 a_{K}^{2}\left|\mathbf{x}-\mathbf{x}_{K}\right|_{2}^{4}
$$

Let

$$
\left(\left.\tilde{u}_{h}\right|_{K}(\mathbf{x})-u_{K}\right)^{2} \leq 2\left(\left|\mathbf{v}_{K}\right|_{2}^{2} h_{K}^{2}+a_{K}^{2} h_{K}^{4}\right) \leq 2\left(\left|\mathbf{v}_{K}\right|_{2}^{2} h^{2}+\frac{h^{4}}{16 \underline{\kappa}^{2}} f_{K}\right),
$$

where we have used the definition of $a_{K}$, the inequality $h_{K} \leq h$, and the assumption

$$
\underline{\kappa} \leq \kappa(\mathbf{x}), \quad \text { a.e. } \quad \mathrm{x} \in \Omega .
$$

Integrating over $K$ and summing over all elements $K$, we obtain

Let

$$
\sum_{K \in \mathcal{M}} \int_{K}\left(\tilde{u}_{h}(\mathbf{x})-u_{K}\right)^{2} d \mathbf{x} \leq 2 h^{2} \sum_{K \in \mathcal{M}} \mathrm{m}(K)\left(\left|\mathbf{v}_{K}\right|_{2}^{2}+\frac{h^{2}}{16 \underline{\kappa}^{2}} f_{K}^{2}\right) .
$$

$$
\begin{aligned}
\left\|\tilde{u}_{h}-u_{h}\right\|_{L^{2}(\Omega)}^{2} & \leq 2 h^{2}\left(\left\|\mathbf{v}_{h}\right\|_{L^{2}(\Omega)^{2}}^{2}+\frac{h^{2}}{16 \underline{\kappa}^{2}}\|f\|_{L^{2}(\Omega)}^{2}\right) \\
& \leq 2 h^{2}\left(\left\|\mathbf{v}_{h}\right\|_{L^{2}(\Omega)^{2}}^{2}+\frac{\operatorname{diam}(\Omega)^{2}}{16 \underline{\kappa}^{2}}\|f\|_{L^{2}(\Omega)}^{2}\right),
\end{aligned}
$$

where $\operatorname{diam}(\Omega)$ is the diameter of the domain $\Omega$. It follows from $[9$, Lemma 4.1] that

$$
\left\|\mathbf{v}_{h}\right\|_{L^{2}(\Omega)^{2}}^{2} \leq \tilde{C}\|f\|_{L^{2}(\Omega)}^{2},
$$

where $\tilde{C}$ is a positive constant independent of the mesh variable $h$. Hence, we obtain the estimation (14).

Next we proof the second estimation (15). Since the Hessian matrix $\mathbf{H}$ of $\tilde{u}_{h}$ satisfies $\mathbf{H}(\mathbf{x})=2 a_{K}, \forall \mathbf{x} \in K$ and $\nabla \tilde{u}_{h}\left(\mathbf{x}_{K}\right)=\mathbf{v}_{K}$, we can apply the mean value theorem as

$$
\left|\nabla \tilde{u}_{h}(\mathbf{x})-\mathbf{v}_{K}\right|_{2} \leq 2\left|a_{K}\right|\left|\mathbf{x}-\mathbf{x}_{K}\right|_{2} \leq 2\left|a_{K}\right| h .
$$

Taking the squared of the above inequality and integrating over $K$ yield

$$
\sum_{K \in \mathcal{M}} \int_{K}\left|\nabla \tilde{u}_{h}(\mathbf{x})-\mathbf{v}_{K}\right|_{2}^{2} d \mathbf{x} \leq \frac{h^{2}}{4 \underline{\kappa}^{2}} \sum_{K \in \mathcal{M}} \mathrm{m}(K) f_{K}^{2},
$$

which implies

$$
\left\|\nabla \tilde{u}_{h}-\mathbf{v}_{h}\right\|_{L^{2}(\Omega)^{2}} \leq \frac{1}{2 \underline{\kappa}}\|f\|_{L^{2}(\Omega)} h .
$$

This completes the proof of the lemma.

Lemma 4.6 (a priori estimation for $\tilde{u}_{h}$ ). Let $u$ be the weak solution of the problem (1) and let $\tilde{u}_{h}$ be the postprocessed solution given by (11). Hence,

$$
\begin{aligned}
\left\|\nabla u-\nabla \tilde{u}_{h}\right\|_{L^{2}(\Omega)^{2}} & \leq C h, \\
\left\|u-\tilde{u}_{h}\right\|_{L^{2}(\Omega)} & \leq C h .
\end{aligned}
$$


Proof. By virtue of triangle inequality, we get

$$
\left\|u-\tilde{u_{h}}\right\|_{L^{2}(\Omega)} \leq\left\|u-u_{h}\right\|_{L^{2}(\Omega)}+\left\|u_{h}-\tilde{u}_{h}\right\|_{L^{2}(\Omega)},
$$

and

$$
\left\|\nabla u-\nabla \tilde{u}_{h}\right\|_{L^{2}(\Omega)^{2}} \leq\left\|\nabla u-\mathbf{v}_{h}\right\|_{L^{2}(\Omega)^{2}}+\left\|\mathbf{v}_{h}-\nabla \tilde{u}_{h}\right\|_{L^{2}(\Omega)^{2}} .
$$

To complete the proof of this lemma it suffices to use the estimations in Lemma 4.1 and Lemma 4.5.

Note that the previous results establish both the convergence of the postprocessed solution $\tilde{u}_{h}$ to the weak solution $u$ of the problem (1) and the convergence of $\nabla \tilde{u}_{h}$ to $\nabla u$ when $h \rightarrow 0$, see for example [22]. However, it should be stressed that to establish these results, some properties on the solution $\tilde{u_{h}}$ are essentially necessary such as the fact that $\tilde{u}_{h}$ and $\nabla \tilde{u}_{h}$ must equal respectively, $u_{K}$ and $\mathbf{v}_{K}$ at $\mathbf{x}_{K}$. As a consequence, the assumption made on the finite volume method in [22, Theorem 3.1] is not used in the analysis presented in this study.

In what follows we present error estimates for our mixed finite volume method. We start by the following result inspired from [21, Lemma 7.1].

Lemma 4.7. For $v, w \in H_{0}^{1}(\Omega)$ and $\tilde{v} \in H^{1}(\mathcal{M})$

$$
\left\|v-\tilde{v}\left|\left\|\leq a\left(v-\tilde{v}, \frac{v-w}{\| v-w|| \mid}\right)+\right\| \tilde{v}-w\right|\right\| .
$$

Proof. Due to the bilinear form of $a(\cdot, \cdot)$ we have

$$
\begin{aligned}
\|v-w \mid\|^{2} & =a(v-\tilde{v}, v-w)+a(\tilde{v}-w, v-w), \\
& \leq\|\tilde{v}-w|\|\times\|||v-w|\|+\||v-w|\| a\left(v-\tilde{v}, \frac{v-w}{\|v-w \mid\|}\right),
\end{aligned}
$$

then,

Hence, if ||$|v-\tilde{v}||| \leq|||v-w|||$, then the proof is completed.

$$
\||v-w|\| \leq\left|\|\tilde{v}-w \mid\|+a\left(v-\tilde{v}, \frac{v-w}{\|\mid v-w\|}\right) .\right.
$$

Let us now consider the case ||$|v-\tilde{v}|\|\geq|||v-w|\|$. In this case,

$$
\begin{aligned}
\|v-\tilde{v}\|^{2} & =a(v-\tilde{v}, v-w)+a(v-\tilde{v}, w-\tilde{v}) \\
& \leq\||v-\tilde{v}||\times|\| w-\tilde{v}|||+\|v-w\|| \mid a\left(v-\tilde{v}, \frac{v-w}{\|v-w \mid\|}\right),
\end{aligned}
$$

and it follows

$$
\begin{aligned}
\|\mid v-\tilde{v}\| \| & \leq\left\||w-\tilde{v} \||+\frac{\||v-w|\|}{\|v-\tilde{v} \mid\|} a\left(v-\tilde{v}, \frac{v-w}{\|v-w\| \mid}\right),\right. \\
& \leq\left\||w-\tilde{v} \||+a\left(v-\tilde{v}, \frac{v-w}{\|v-w \mid\|}\right),\right.
\end{aligned}
$$

which resumes the proof.

We use the Oswald's interpolation operator introduced in [21]

$$
\mathcal{I}_{\mathrm{Os}}: P^{2}(\mathcal{M}) \longrightarrow P^{2}(\mathcal{M}) \cap H^{1}(\Omega),
$$

defined as $\forall v_{h} \in H^{1}(\mathcal{M})$,

$$
\mathcal{I}_{\mathrm{Os}}\left(v_{h}\right)(P)=\left\{\begin{array}{lll}
\left.\frac{1}{\operatorname{Card}\left(\mathcal{V}_{\mathrm{P}}\right)} \sum_{K \in \mathcal{V}_{P}} v_{h}\right|_{K}(P), & \text { if } & P \in \mathcal{S}, \\
\left.\left\{v_{h}\right\}\right|_{\sigma}(P), & \text { if } \quad P=\mathbf{x}_{\sigma},
\end{array}\right.
$$


where

$$
\left.\left\{v_{h}\right\}\right|_{\sigma}=\left\{\begin{array}{llrl}
\frac{1}{2}\left(\left.v_{h}\right|_{K}+\left.v_{h}\right|_{L}\right), & \text { if } & \sigma=K \mid L, \\
\left.v_{h}\right|_{K}, & \text { if } & \sigma \in \mathcal{E}_{K} \cap \mathcal{E}_{\text {ext }} .
\end{array}\right.
$$

Lemma 4.8 (Estimation of the upper bound). Let $u$ be the weak solution of the problem (1) and let $\tilde{u}_{h}$ be the postprocessed solution given by (11). Hence,

$$
\left\|\mid u-\tilde{u}_{h}\right\| \| \leq\left\{\left(\sum_{K \in \mathcal{M}} \eta_{\mathrm{R}, K}\left(\tilde{u}_{h}\right)^{2}\right)^{1 / 2}+\left(\sum_{K \in \mathcal{M}} \eta_{\mathrm{NC}, K}\left(\tilde{u}_{h}\right)^{2}\right)^{1 / 2}\right\}
$$

with

$$
\begin{array}{rlrl}
\eta_{\mathrm{R}, K}\left(\tilde{u}_{h}\right):=h_{K} \sqrt{\frac{C_{P, K}}{\kappa_{K}}}\left\|f+\nabla \cdot\left(\left.\kappa_{K} \nabla \tilde{u}_{h}\right|_{K}\right)\right\|_{K}, & & \forall K \in \mathcal{M}, \\
\eta_{\mathrm{NC}, K}\left(\tilde{u}_{h}\right):=\left\|\tilde{u}_{h}-\mathcal{I}_{\mathrm{Os}}\left(\tilde{u}_{h}\right)\right\|_{K}, & \forall K \in \mathcal{M} .
\end{array}
$$

Proof. Let $\phi$ be a function in $H_{0}^{1}(\Omega)$. Due to the bilinear form of $a(\cdot, \cdot)$ and the Green theorem, we have

$$
\begin{aligned}
a\left(u-\tilde{u}_{h}, \phi\right) & =(f, \phi)_{\Omega}-\sum_{K \in \mathcal{M}}\left(\left.\kappa_{K} \nabla \tilde{u}_{h}\right|_{K}, \nabla \phi\right)_{K}, \\
& =\sum_{K \in \mathcal{M}}\left(f+\nabla \cdot\left(\left.\kappa_{K} \nabla \tilde{u}_{h}\right|_{K}\right), \phi\right)_{K}-\sum_{K \in \mathcal{M}}\left(\left.\kappa_{K} \nabla \tilde{u}_{h}\right|_{K} \cdot \mathbf{n}, \phi\right)_{\partial K} .
\end{aligned}
$$

Let us consider the term $\sum_{K \in \mathcal{M}}\left(\left.\kappa_{K} \nabla \tilde{u}_{h}\right|_{K} \cdot \mathbf{n}, \phi\right)_{\partial K}$ which we denote by $A$. Thus,

$$
A=\sum_{K \in \mathcal{M}} \sum_{\sigma \in \mathcal{E}_{K}}\left(\left.\kappa_{K} \nabla \tilde{u}_{h}\right|_{K} \cdot \mathbf{n}_{K, \sigma}, \phi\right)_{\sigma}
$$

If we assemble by interfaces, the term $A$ can be reformulated as

$$
A=\sum_{\substack{\sigma \in \mathcal{E}_{\text {int }} \\ \sigma=K \mid L}}\left(\left.\kappa_{K} \nabla \tilde{u}_{h}\right|_{K} \cdot \mathbf{n}_{K, \sigma}+\left.\kappa_{L} \nabla \tilde{u}_{h}\right|_{L} \cdot \mathbf{n}_{L, \sigma}, \phi\right)_{\sigma},
$$

where we have used the fact that $\phi$ vanishes on the boundary.

From the equation (10c) we have

$$
\left.\kappa_{K} \nabla \tilde{u}_{h}\right|_{K} \cdot \mathbf{n}_{K, \sigma}+\left.\kappa_{L} \nabla \tilde{u}_{h}\right|_{L} \cdot \mathbf{n}_{L, \sigma}=\frac{1}{\mathrm{~m}(\sigma)}\left(F_{K, \sigma}+F_{L, \sigma}\right) .
$$

By virtue of (3a) one obtains

$$
A=0,
$$

and therefore,

$$
a\left(u-\tilde{u}_{h}, \phi\right)=\sum_{K \in \mathcal{M}}\left(f+\nabla \cdot\left(\left.\kappa_{K} \nabla \tilde{u}_{h}\right|_{K}\right), \phi\right)_{K} .
$$

We set $\phi_{K}=\frac{1}{\mathrm{~m}(\mathrm{~K})}(\phi, 1)_{K}$. using the definition $(3)$ of the mixed finite volume method and the equation (10c) we obtain

This yields

$$
\left(f+\nabla \cdot\left(\left.\kappa_{K} \nabla \tilde{u}_{h}\right|_{K}\right), \phi_{K}\right)_{K}=0
$$

$$
a\left(u-\tilde{u}_{h}, \phi\right)=a\left(u-\tilde{u}_{h}, \phi-\phi_{K}\right)
$$


Hence,

$$
\begin{aligned}
a\left(u-\tilde{u}_{h}, \phi\right) & \leq \sum_{K \in \mathcal{M}}\left\|f+\nabla \cdot\left(\left.\kappa_{K} \nabla \tilde{u}_{h}\right|_{K}\right)\right\|_{K}\left\|\phi-\phi_{K}\right\|_{K}, \\
& \leq \sum_{K \in \mathcal{M}} \eta_{\mathrm{R}, K} \kappa_{K}^{1 / 2}\|\nabla \phi\|_{K} .
\end{aligned}
$$

By setting $\phi=\frac{u-\mathcal{I}_{\mathrm{Os}}\left(\tilde{u}_{h}\right)}{\left\|u-\mathcal{I}_{\mathrm{Os}}\left(\tilde{u}_{h}\right)\right\|}$ in the above inequality and using the inequality (16) we obtain

$$
\begin{aligned}
\left\|\mid u-\tilde{u}_{h}\right\| \| & \leq\left(\sum_{K \in \mathcal{M}} \eta_{\mathrm{R}, K}\left(\tilde{u}_{h}\right)^{2}\right)^{1 / 2}+\left\|\tilde{u}_{h}-\mathcal{I}_{\mathrm{Os}}\left(\tilde{u}_{h}\right)\right\| \\
& \leq\left\{\left(\sum_{K \in \mathcal{M}} \eta_{\mathrm{R}, K}\left(\tilde{u}_{h}\right)^{2}\right)^{1 / 2}+\left(\sum_{K \in \mathcal{M}} \eta_{\mathrm{NC}, K}\left(\tilde{u}_{h}\right)^{2}\right)^{1 / 2}\right\}
\end{aligned}
$$

which achieves the proof.

Notice that an estimation of the lower bound can be obtained for the proposed mixed finite volume method using the same ideas reported in [21, Theorem 4.4].

\section{Numerical Results}

In this section, we present numerical results for two test examples to support our a posteriori error estimates. We solve the two-dimensional diffusion problem with high heterogeneous diffusion coefficient $\kappa(\mathbf{x})$ defined as

$$
\begin{array}{rlrl}
-\nabla \cdot(\kappa(\mathbf{x}) \nabla u(\mathbf{x})) & =f(\mathbf{x}), & & \forall \mathbf{x} \in \Omega, \\
u(\mathbf{x})=g(\mathbf{x}), & & \forall \mathbf{x} \in \partial \Omega,
\end{array}
$$

where $f$ and $g$ are determined from a given analytical solution in polar coordinates, compare [21, 22] among others. In both examples the numerical solution presents a singularity at the origin of the computational domain $\Omega$. Mesh adaptation based on the proposed error estimates is applied to get a suitable locally refined mesh for triangular elements.

The mesh generation is based on the Delaunay triangulation using the software Triangle developed in [19]. Starting from a coarse initial mesh, we consider both uniform and adaptive refinements using a procedure based in our a posteriori estimators. The adaptive refinement consists of refining each triangle where the local value of the estimated error is greater than the half of the maximum of the global estimator. We also denote by DoF the number of elements in the considered mesh, by $f_{\eta}$ the effective index defined as the ratio between the related estimator and the true error, and by $\epsilon$ the energy error. In all the computations reported herein, the linear systems of algebraic equations are solved using the BiCGSTAB solver. In addition, all stopping criteria for iterative solvers were set to $10^{-9}$, which is small enough to guarantee that the algorithm truncation error dominates the total numerical error.

5.1. Example 1. We solve the diffusion problem (18) in the squared domain $\Omega=[-1,1]^{2}$ divided in four subdomains $\Omega_{i}(i=1, \ldots, 4)$ as shown in Figure 1 . The diffusion coefficient $\kappa$ is constant in each subdomain $\left(\left.\kappa\right|_{\Omega_{i}}=\kappa_{i}\right.$, $i=1, \ldots, 4)$ and functions $f$ and $g$ are selected such that the exact solution $u$ in polar coordinates $(r, \theta)$ is

$$
\left.u\right|_{\Omega_{i}}(r, \theta)=r^{\alpha}\left(a_{i} \sin (\alpha \theta)+b_{i} \cos (\alpha \theta)\right), \quad i=1, \ldots, 4,
$$

where $\alpha$ is a fixed parameter in our simulations, $a_{i}$ and $b_{i}$ are constants related to the subdomains $\Omega_{i}$.

In the first run we use the following parameters

$$
a_{1}=0.44721360, \quad a_{2}=-0.74535599, \quad a_{3}=-0.94411759, \quad a_{4}=-2.40170264,
$$




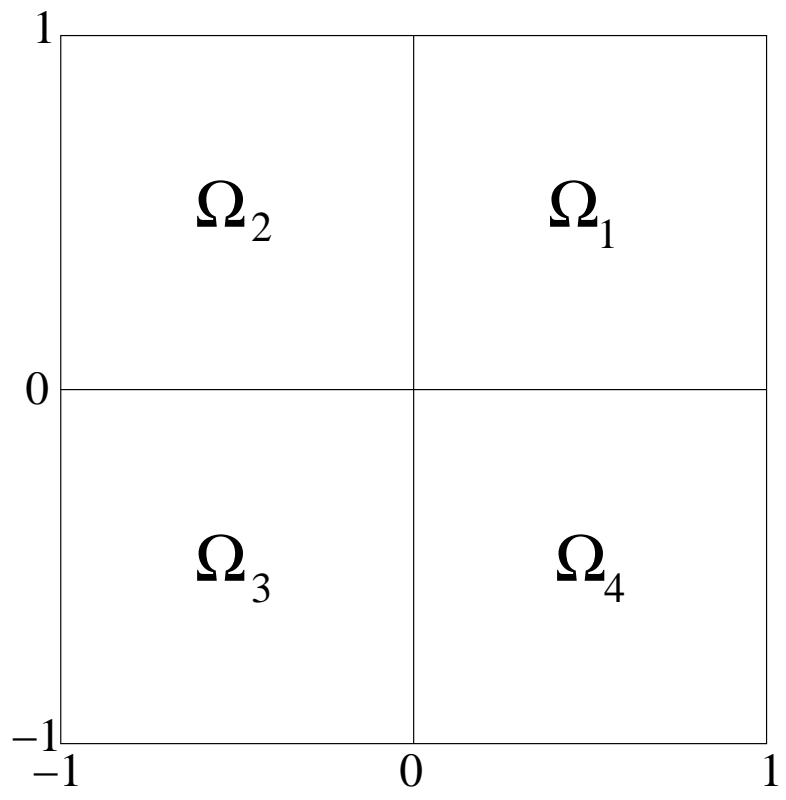

Figure 1. Domain configuration for Example 1.

$$
b_{1}=1, \quad b_{2}=2.96039604, \quad b_{3}=0.55555556, \quad b_{4}=-0.48148148,
$$

$\alpha=0.53544095$ and the diffusion coefficients are given as

$$
\kappa_{1}=\kappa_{3}=5, \quad \kappa_{2}=\kappa_{4}=1
$$

Figure 2 shows the adaptive meshes, numerical solutions, exact solutions, estimated errors and absolute errors at initial and two different iterations. As can be seen from the results presented in this figure, no noticeable differences are visible between the exact and mixed finite volume solutions. However, in term of the estimated and absolute errors, the difference can be clearly seen on the coarse mesh whereas, this difference becomes negligible as the mesh is refined in the later iterations. It is clear that the proposed error estimator locates the exact error very well and the maximum error located at the center of the computational domain is well captured. In addition, for the considered ratio between the diffusion coefficients $\kappa_{1}$ and $\kappa_{2}$, we can observe that the grid is highly refined around the domain center, which confirms the relevance of the adaption criteria based on our estimator.

In Figure 4 we display the accuracy and efficiency plots as a function of number of DoFs. The left plot in Figure 4 compares the estimated energy errors using both uniform and adaptive mesh refinements. The efficiency of the mixed finite volume method is quantified by plotting the effective index versus the number of DoFs in the right plot in Figure 4. For a better insight logarithmic scales are used in these plots. It is clear that for a given precision, the adaptive refinement allows to reduce consequently the number of unknowns in comparison with the uniform adaption. In addition, we note that both uniform refinements and adaptive refinements get good approximate solutions as the number of DoFs increases, and the convergence orders are nearly optimal. However, to obtain similar accuracy, the adaptive refinements will use less control volumes. It should also be noted that the efficiency plot shows a numerical asymptotic exactness of our estimator.

Next we consider the test case with the following parameters

$$
\begin{aligned}
& a_{1}=0.1, \quad a_{2}=-9.60396040, \quad a_{3}=-0.480354870, \quad a_{4}=7.70156488, \\
& b_{1}=1.0, \quad b_{2}=2.96039604, \quad b_{3}=-0.88275659, \quad b_{4}=-6.45646175,
\end{aligned}
$$

$\alpha=0.12690207$ and the diffusion coefficients are set to

$$
\kappa_{1}=\kappa_{3}=100, \quad \kappa_{2}=\kappa_{4}=1 .
$$



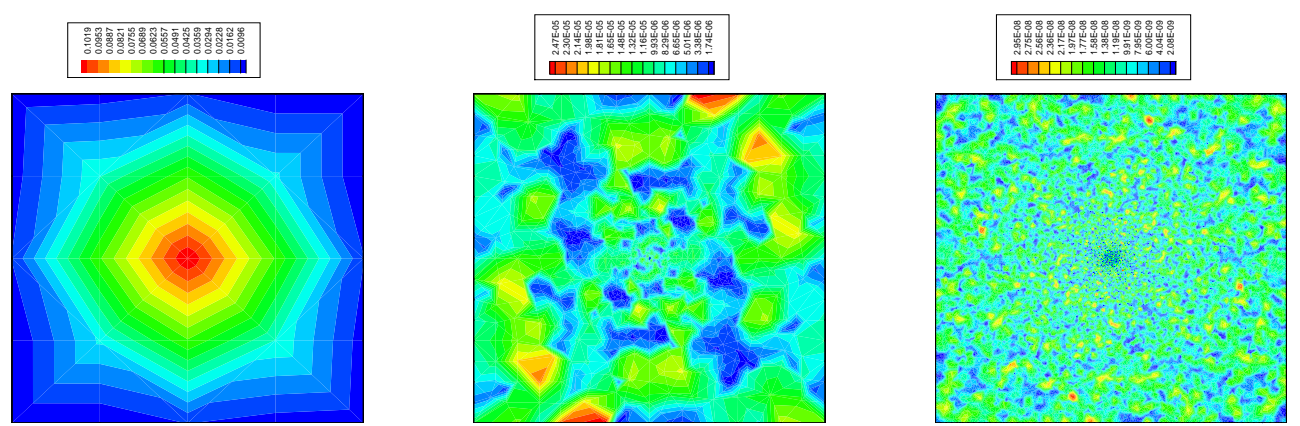

్ㅣㅁ
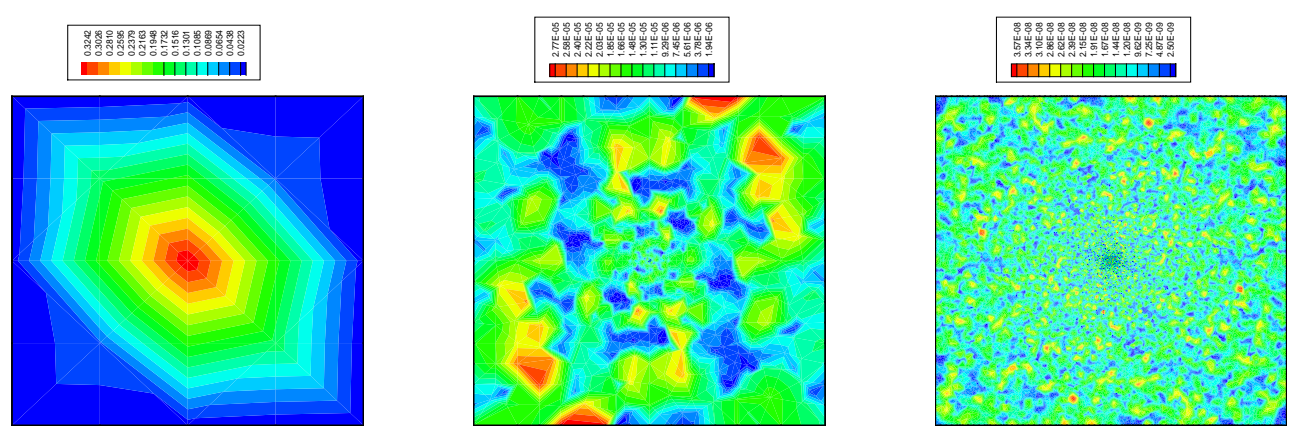

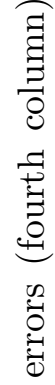

足

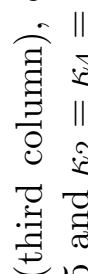
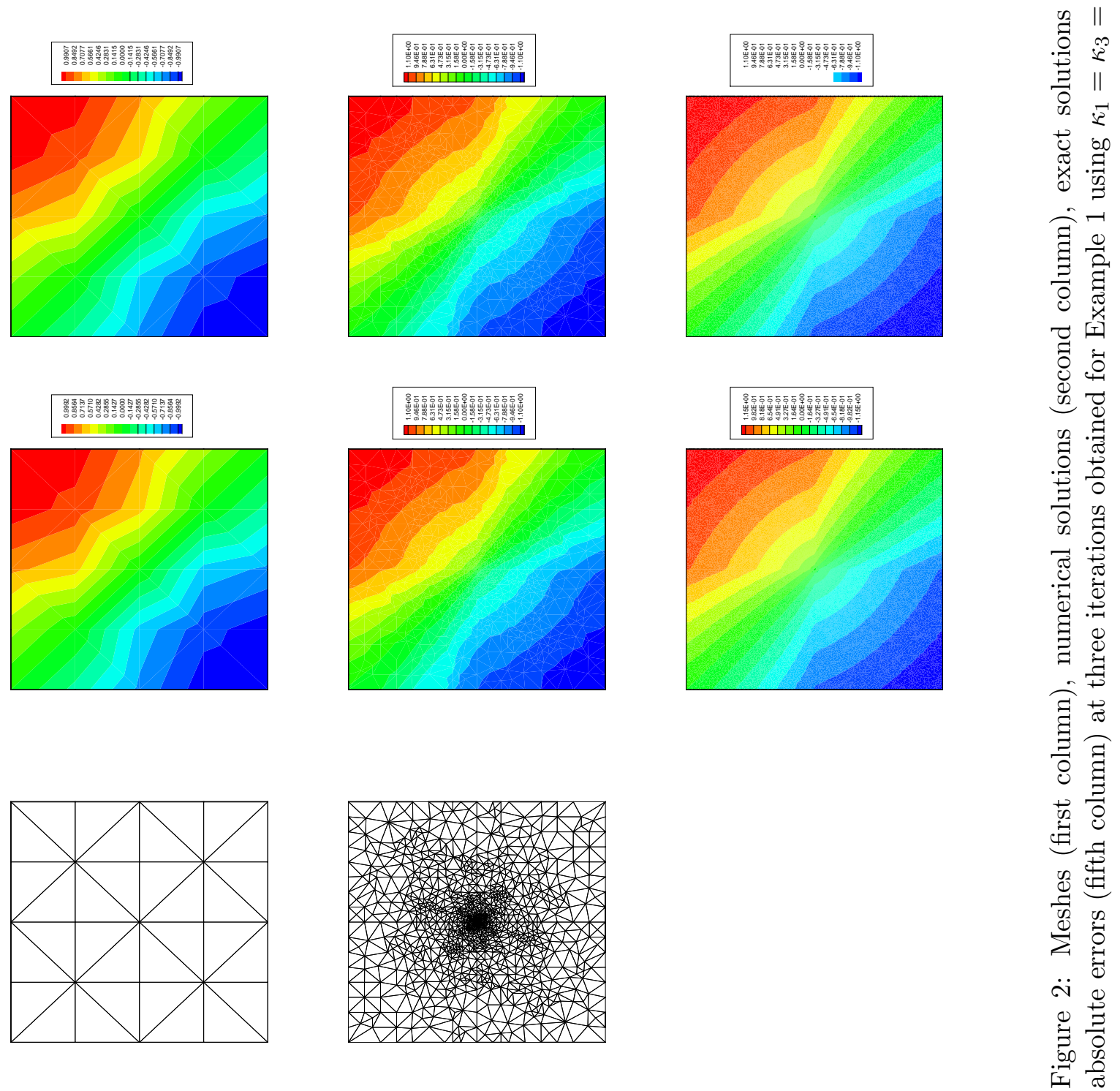

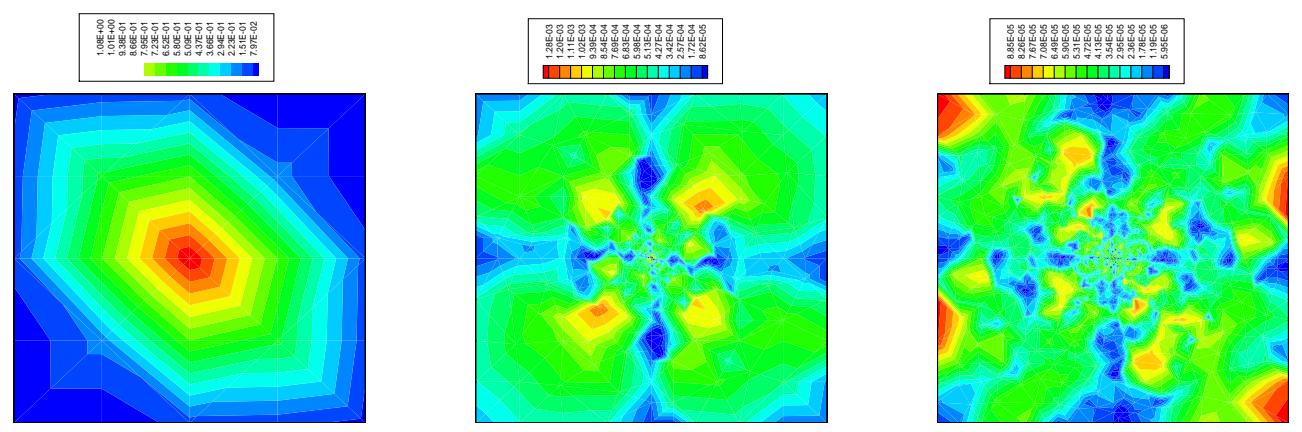

్ㅣㅁ
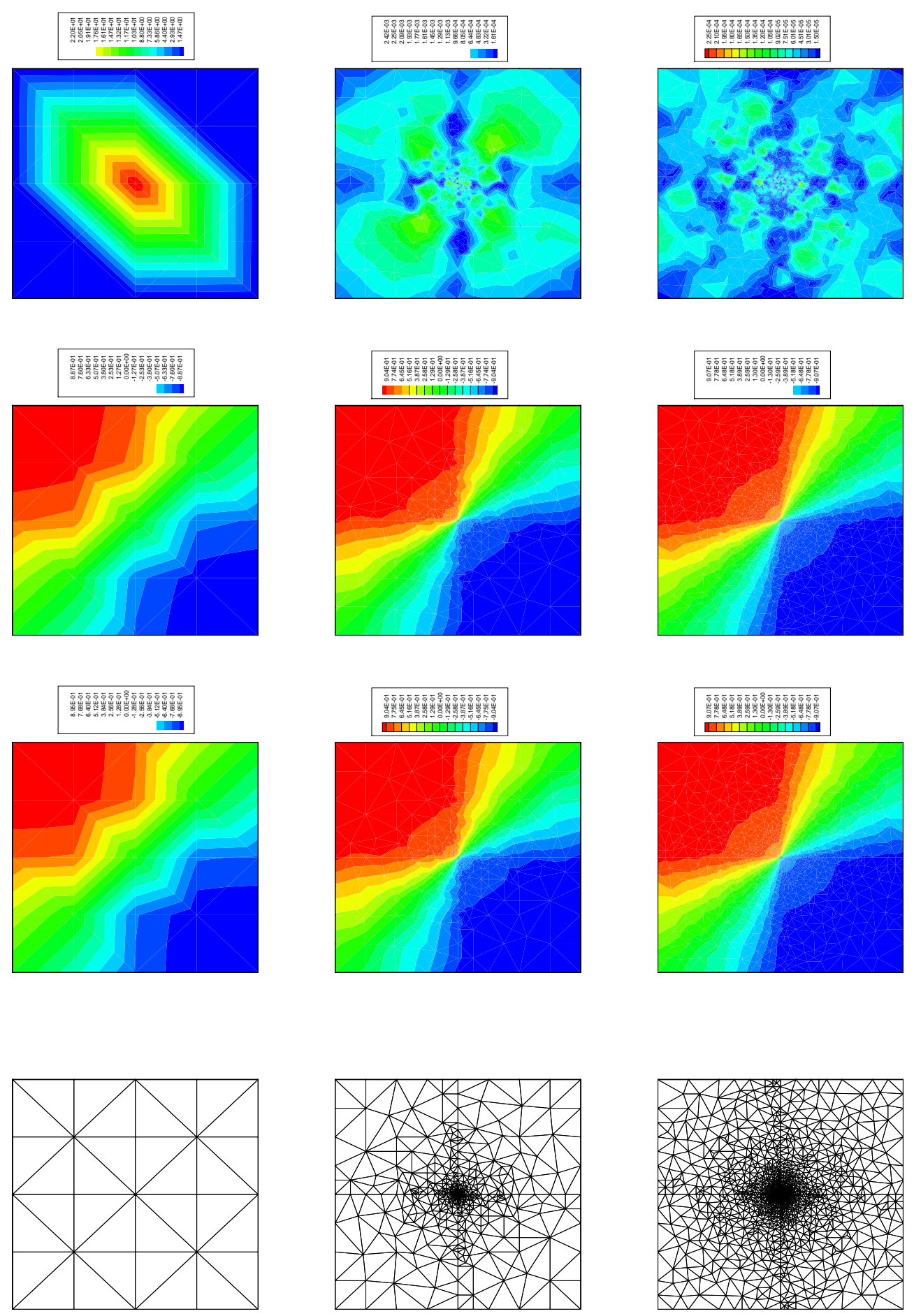

పี

总

.

吾

苟

范

몰

总

青.

क

శึ่

忌

声

$\exists$

专

킹

잉

잉

تే

过

常

$\stackrel{0}{\vdots}$

ले

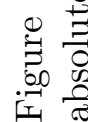



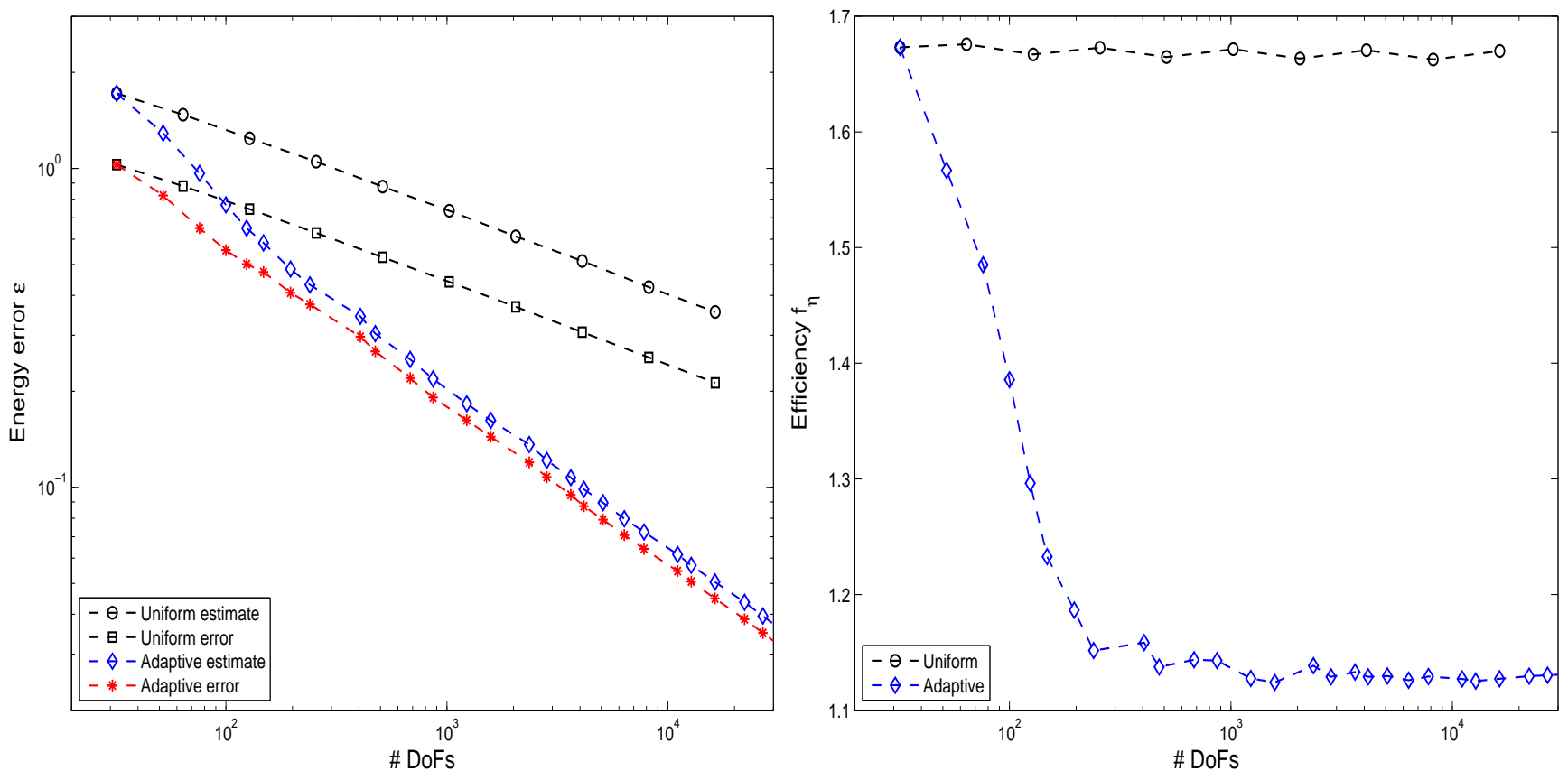

FiguRE 4. Accuracy results (left plot) and efficiency results (right plot) as a function of number of DoFs obtained for Example 1 using $\kappa_{1}=\kappa_{3}=5$ and $\kappa_{2}=\kappa_{4}=1$.
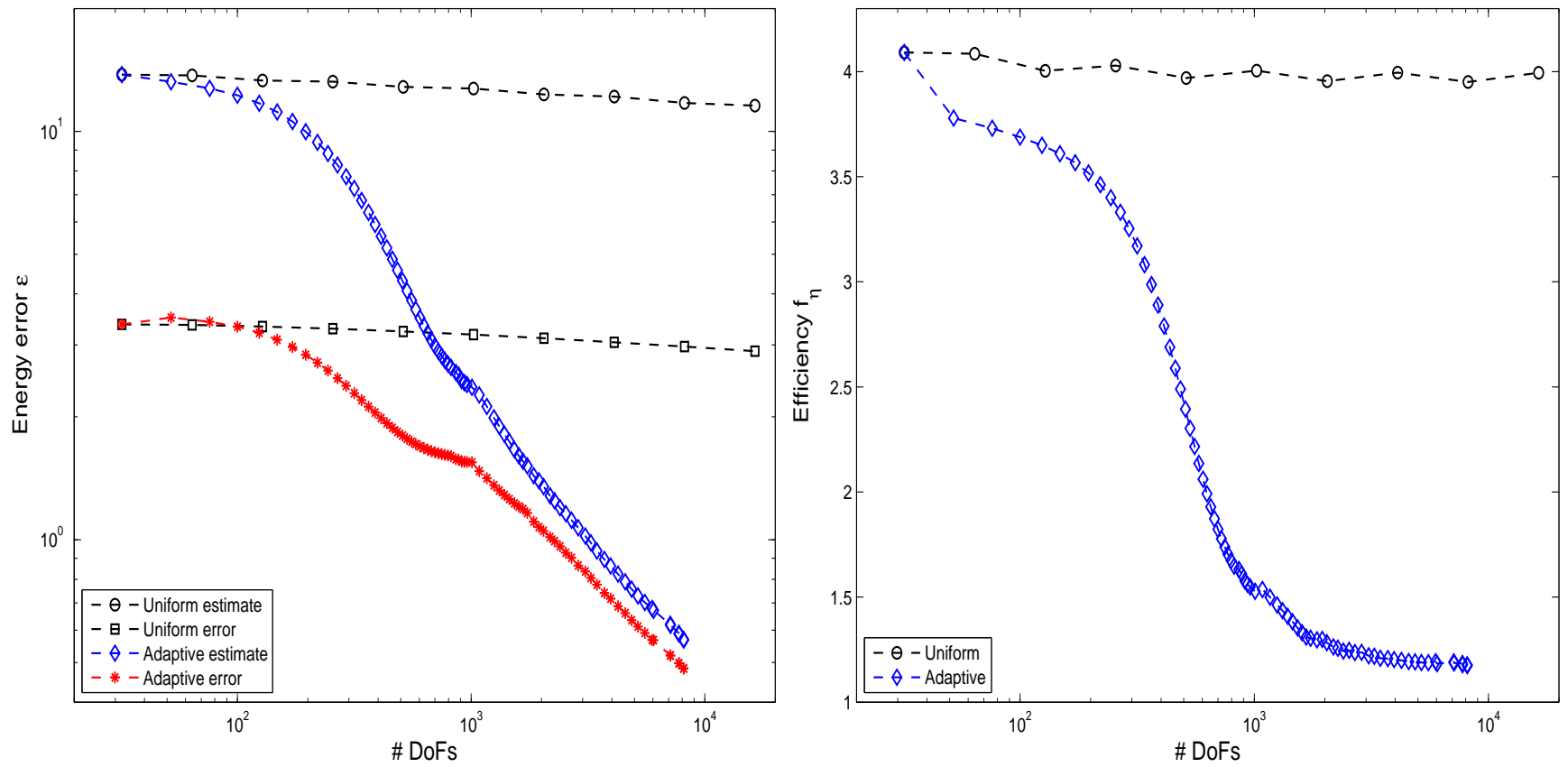

FiguRE 5. Accuracy results (left plot) and efficiency results (right plot) as a function of number of DoFs obtained for Example 1 using $\kappa_{1}=\kappa_{3}=100$ and $\kappa_{2}=\kappa_{4}=1$. 
Note that in this test case, the diffusion ratio $\kappa_{1} / \kappa_{2}$ is 20 times larger than in the previous test case which results in a stronger singularity at the domain center. The exact and computed results are displayed in Figure 3. As expected the numerical solutions show strong singularity at the center of the computational domain which is underlined by the mesh refinements in this region of the domain. Compared to the previous test case $\left(\kappa_{1} / \kappa_{2}=5\right)$, the mesh refinement is mainly concentrated in the center of the domain, compare the mesh configurations after the adaptive refinements in Figure 3. It should also be noted that the discrepancy between the true and estimated errors is remarkably small as compared to the magnitude of the target functional. The distribution of error contributions reflects the qualitative behavior of local errors and indicates that stronger mesh refinement is required in the vicinity of domain center as the diffusion ratio $\kappa_{1} / \kappa_{2}$ increases.

The accuracy and efficiency results for this example are depicted in Figure 5. In the very beginning of iterations, the uniform and estimated errors are similar; however, we notice that the errors decrease generally during the evolving mesh adaptations while on the uniform grid the errors are evolved constantly. In spite of a large number of control volumes, the uniform mesh without refinement at the singularity location appears disqualified when the singularity is present after iterations; on the contrary, the established a posteriori error estimator can follow the development of the singularity and, thus, guide the grid well adapted to physical solution behavior. This comparison reveals the necessity of using mesh adaptation coupled with a posteriori error estimate analysis for sophisticated diffusion simulation quality enhancement. As we show for the considered diffusion problem, this procedure allows us to reach significant improvement in accuracy and stability, compared to the uniform adaptation, within a small number of iterations.

5.2. Example 2. The second example consists of solving the diffusion problem (18) in a radial plane domain divided in four subdomains $\Omega_{i}(i=1, \ldots, 4)$ as shown in Figure 6 . As in the previous example, the source functions $f$ and $g$ are selected such that (19) is the exact solution of the problem in the polar coordinates $(r, \theta)$. Here, the parameters $\alpha$, the constants $a_{i}$ and $b_{i}$, and the diffusion coefficients $\kappa_{i}$ are selected the same as in the first run of Example 1. The main aim of this example is to check the performance of the proposed mixed finite volume method for solving heterogeneous diffusion problems in relatively complex domains. The obtained results are reported in Figure 7 in same manner as in the previous example. Again there is a good agreement between the predicted error with the exact one. Our numerical simulations demonstrate that the coupling of mesh adaptation and a posteriori error estimate allows for an economical and accurate finite volume solution of diffusion equations with singularities in their solutions.

In Figure 8 we illustrate the evolution of the energy error and effective index as a function of the number of DoFs. Similar conclusions to the previous simulations in the squared domain can be drawn for this test example. We can observe, that while the regions of higher error are decreasing for the estimated method when refining the mesh, they remain almost independent of refinement for the uniform method. We attribute this phenomenon to the geometry of the domain.

\section{Conclusions}

We have developed a posteriori error analysis for mixed finite volume methods for solving second-order elliptic equations with a scalar diffusion tensor. We have derived an efficient and effective adaptive strategy based on fully computable error estimators. Global upper and lower bounds are proved, and the considered test examples show that both error estimators are easy to compute. Indeed no local problems are solved and we only use the values provided by the finite volume scheme. It follows that our scheme would be suitable for coupled elliptic/hyperbolic equations on unstructured meshes. To assess the validity and accuracy of the proposed mixed finite volume method, it is applied to solve two specific problems with known analytical solutions. The numerical results obtained agree favorably with the known solutions indicating that the method can be used to provide reliable and accurate numerical solutions for diffusion equations in heterogeneous media. Future work will concentrate on extending this analysis to second-order elliptic equations with fully diffusion tensor. 


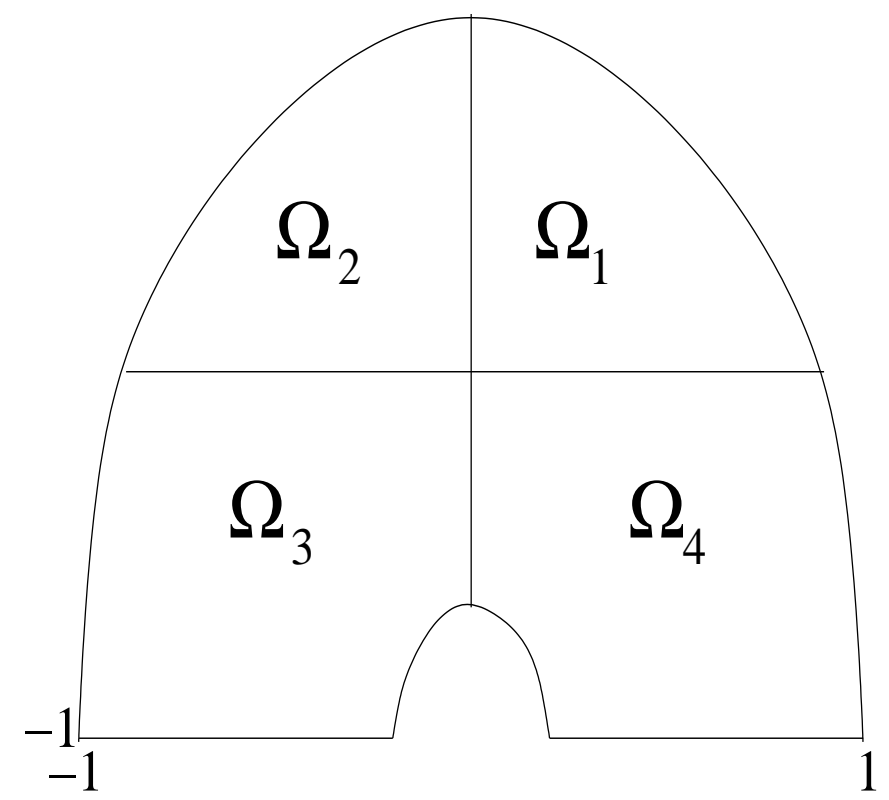

Figure 6. Domain configuration for Example 2.

\section{Acknowledgments}

The second and third authors acknowledge the support from the Université Paris 13 in France for their visit to LAGA group during which, part of this work was performed.

\section{References}

[1] I. Aavatsmark, T. Barkve, O. Boe, and T. Mannseth. Discretization on unstructured grids for inhomogeneous anisotropic media part1: Derivation of the methods. SIAM, Journal of scientific computing, 19(5):1700-1716, 1998.

[2] L. Anh Ha and P. Omnes. An a posteriori error estimation for the discrete duality finite volume discretization of the stokes equations. ESAIM: M2AN, 49:663-693, 2015.

[3] I. Babuška and W.C. Rheinbold. Error estimates for adaptive finite element computations. SIAM J. Numer. Anal., 15:736-754, 1978.

[4] C. Chainais-Hillairet and J. Droniou. Convergence analysis of a mixed finite volume scheme for an ellipticparabolic system modeling miscible fluid flows in porous media. SIAM, J. Numer. Anal., 45:2228-2258, 2007.

[5] C. Chen, Y. Chen, and X. Zhao. A posteriori error estimates of two-grid finite volume element methods for nonlinear elliptic problems. Computers \&S Mathematics with Applications, 75:1756-1766, 2018.

[6] S. Cochez-Dhont, S. Nicaise, and S. Repin. A posteriori error estimates for finite volume approximations. Math. Model. Nat. Phenom., 4:106-122, 2009.

[7] L. B. Da Veiga and G. Manzini. An a posteriori error estimator for the mimetic finite difference approximation of elliptic problems. Comput. Methods Appl. Mech. Engrg, 76:1696-1723, 2008.

[8] K. Domelevo and P. Omnes. A finite volume method for the laplace equation on almost arbitrary twodimensional grids. ESAIM, Math. Mod. Numer. Anal., 39(6):1203-1249, 2005.

[9] J. Droniou and R. Eymard. A mixed finite volume scheme for anisotropic diffusion problems on any grid. Numer. Math., 105:35-71, 2006. 

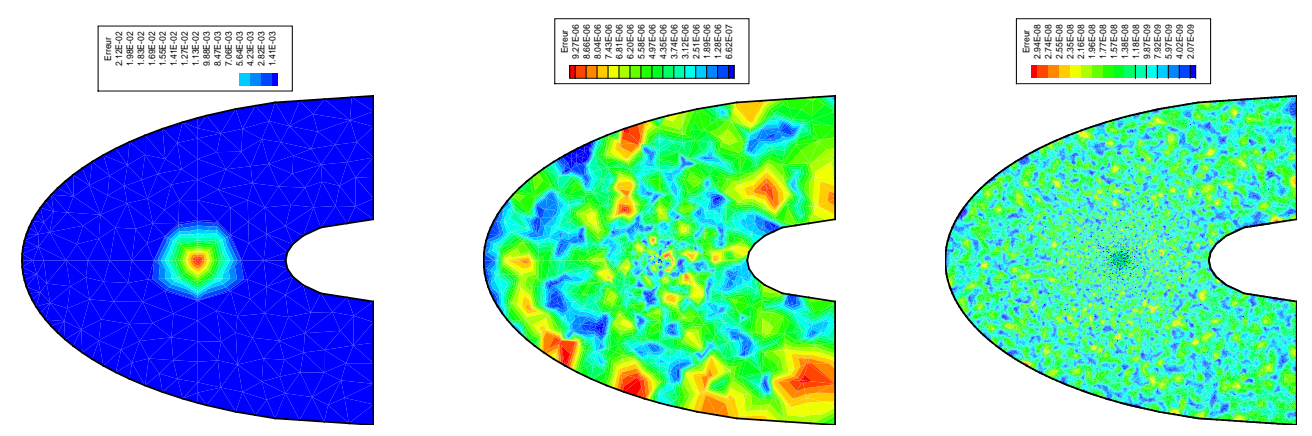

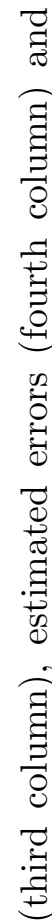
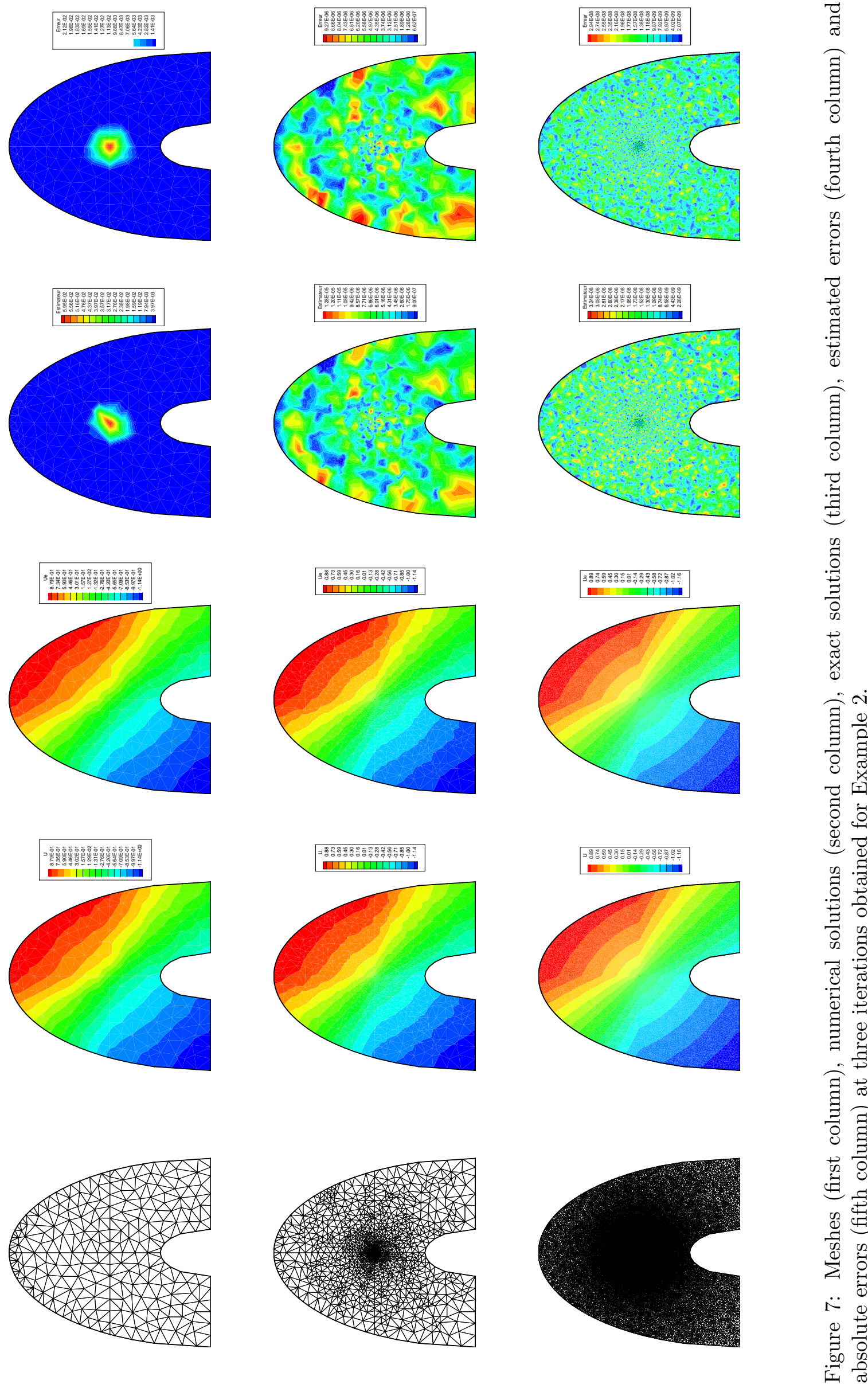

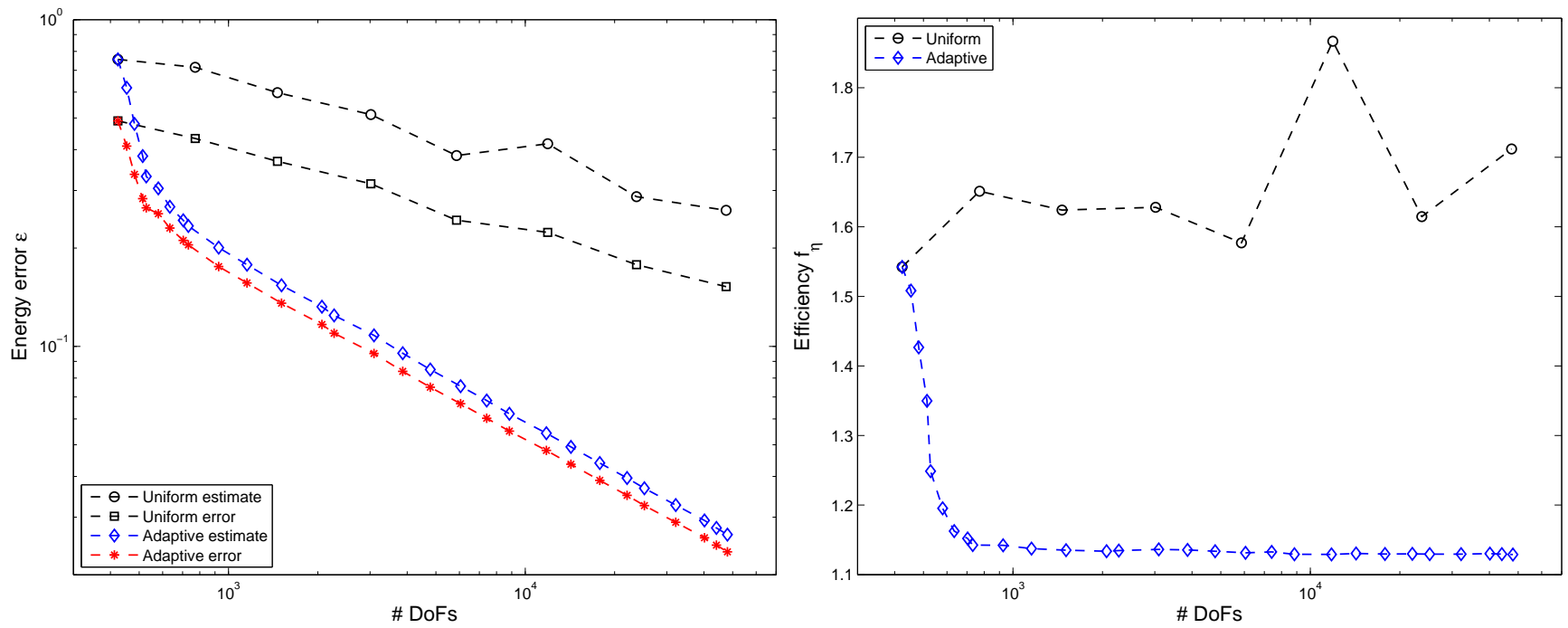

FiguRE 8. Accuracy results (left plot) and efficiency results (right plot) as a function of number of DoFs obtained for Example 2.

[10] J. Droniou, R. Eymard, T. Gallouët, and R. Herbin. A unified approach to mimetic finite difference, hybrid finite volume and mixed finite volume methods. Math. Model. Meth. Appl. Sci., 20(2):265-295, 2010.

[11] J. Droniou, R. Eymard, T. Gallouët, and R. Herbin. Gradient schemes: a generic framework for the discretization of linear, nonlinear and nonlocal elliptic and parabolic equations. Math. Model. Meth. Appl. Sci., 23(13):2395-2432, 2013.

[12] R. Eymard, T. Gallouët, and R. Herbin. Finite volume approximation of elliptic problems and convergence of approximate gradient. Applied Numerical Mathematic, 37:31-53, 2001.

[13] H. Hakula, M. Neilan, and J.S. Ovall. A posteriori estimates using auxiliary subspace techniques. Journal of Scientific Computing, 72:97-127, 2017.

[14] F. Hermeline. A finite volume method for approximating 3D diffusion operators on general meshes. J. Comput. Phys., 228:5763-5786, 2009.

[15] A. Mahamane. Analysis of the mixed finite volume scheme for a convection-diffusion equation. Finite Volumes for Complex Applications R. Eymard and J.-M. Hérard (eds), V:569-576, 2008.

[16] S. Nicaise. A posteriori error estimations of some cell centered finite volume methods for convection- diffusionreaction problems. SIAM, J. Numer. Anal., 44:949-978, 2006.

[17] P. Omnes, Y. Penel, and Y. Rosenbaum. A posteriori error estimation for the discrete duality finite volume discretization of the the laplace equation. SIAM, J. Numer. Anal., 47:2782-2807, 2009.

[18] R. Herbin R. Eymard, T. Gallouët. Finite Volume Methods, Handbook of Numerical Analysis. Elsevier Science, 2000.

[19] J.R. Shewchuk. Triangle Engineering a 2D quality mesh generator and delaunay triangulator in Applied Computational Geometry: Towards Geometric Engineering Lecture Notes in Comput. Sci. 1148, M. C. Lin and D. Manocha, eds. Springer-Verlag, Berlin, 1996.

[20] R. Verfurth. A Review of A Posteriori Error Estimation and Adaptive Mesh-Refinement Techniques. Wiley/Teubner, Stuttgart, 1996.

[21] M. Vohralík. A posteriori error estimates for lowest-order mixed finite element discretizations of convectiondiffusion-reaction equations. SIAM, J. Numer. Anal., 45:1570-1599, 2007.

[22] M. Vohralík. Residual flux-based a posteriori error estimates for finite volume discretizations of inhomogeneous, anisotropic, and convection-dominate problems. Numer. Math., 1:121-158, 2008. 